\title{
Fault Identification, Diagnosis, and Prognostics Based on Complex Signal Analysis
}

\author{
Minvydas Ragulskis $\left(\mathbb{D},{ }^{1} \text { Chen Lu }(\mathbb{D})^{2} \text { Maosen Cao } \mathbb{D}\right)^{3}$ \\ Gangbing Song $\left(\mathbb{i},{ }^{4}\right.$ and Rafal Burdzik $\oplus^{5}$ \\ ${ }^{1}$ Center for Nonlinear Systems, Kaunas University of Technology, Kaunas, Lithuania \\ ${ }^{2}$ School of Reliability and Systems Engineering, Beihang University, Beijing, China \\ ${ }^{3}$ Institute of Vibration and Dynamics, Hohai University, Nanjing, China \\ ${ }^{4}$ Department of Mechanical Engineering, University of Houston, Houston, USA \\ ${ }^{5}$ Faculty of Transport, Silesian University of Technology, Katowice, Poland
}

Correspondence should be addressed to Minvydas Ragulskis; minvydas.ragulskis@ktu.lt

Received 21 October 2018; Accepted 6 December 2018; Published 17 December 2018

Copyright (C) 2018 Minvydas Ragulskis et al. This is an open access article distributed under the Creative Commons Attribution License, which permits unrestricted use, distribution, and reproduction in any medium, provided the original work is properly cited.

This special issue titled "Fault Identification, Diagnosis, and Prognostics Based on Complex Signal Analysis" is a truly interdisciplinary issue. Manuscripts published in this special issue do represent such diverse fields as mechanical engineering, aviation engineering and technology, electric and electronic engineering, statistics, and so on. The geography of authors spans over three continents, Asia, Europe, and America. A total of 18 manuscripts were published in this issue.

This special issue gathered several studies that focus on fault detection and diagnosis of mechanical products, such as bolts, rolling bearings, gears, and hydraulic servo systems. For example, G. Wu et al. proposed a modified time reversal method and successfully realized bolt loosening detection and localization in simulated thermal protection system panels. Two studies aim to reduce the background noise in monitoring signal of rolling bearings, thus improving the effectiveness of fault diagnosis. R. Yuan et al. reported an adaptive high-order local projection denoising method and demonstrated the characteristic frequencies of simulated signals can be well extracted by the proposed method. D. Zhong et al. proposed a novel fault signal denoising scheme based on improved sparse regularization via convex optimization to extract the fault feature of rolling bearing. Experiments show that the proposed method has a better performance than traditional methods. In an interesting study, a method based on the multiscale chirplet path pursuit and the linear canonical transform is proposed by X. Shuiqing et al. and applied to diagnose the gear fault in the variable speed condition for the first time. This method can diagnose the gear faults availably. In another study, Y. Ding et al. presented a fault diagnosis scheme for hydraulic servo system using compressed random subspace based ReliefF (CRSR) method. The proposed CRSR method is able to enhance the robustness of the feature information against interference while selecting the feature combination with balanced information expressing ability. Finally, dealing with sliding mode Fault Tolerant Control (FTC) problem for nonlinear system, two adaptive sliding mode FTC schemes for an uncertain nonlinear system subject to multiplicative and process faults were presented by A. Ben Brahim et al. By solving a single-step multiobjective LMI optimization problem, the observer and controller gains are obtained, offering a solution to stabilize the closed-loop nonlinear system despite the occurrence of real fault effects.

This special issue also attracted several studies from the field of aviation engineering and technology. H. Liu et al., for example, proposed a fault diagnosis method for electromechanical actuators (EMAs) based on variational mode decomposition (VMD) multifractal detrended fluctuation analysis (MFDFA) and probabilistic neural network 
(PNN). This method is demonstrated to achieve effective fault diagnosis for EMAs under different working conditions. In another elegant study, a deep learning approach is proposed by J. Ma et al. to predict the RUL of an aircraft engine based on a stacked sparse autoencoder and logistic regression. The proposed method also has significance for enhancing the safety of aircraft engines and prognosticating and managing the health of aircraft engines to reduce the cost of maintenance. In addition, as an interdisciplinary method, Y. Cheng et al. introduced visual cognition theory into the field of lithium-ion battery capacity estimation. Inspired by multichannel and manifold sensing characteristics of human visual system, battery capacity is effectively estimated based on nonsubsampled contourlet transform and Laplacian eigenmap manifold learning method.

Two studies in this special issue are related to electric and electronic engineering. D. Song et al. reported a general fault detection and diagnosis scheme based on observers and residual error analysis for superheterodyne receivers. The proposed method may provide a promising approach not only to the superheterodyne receiver but also to more complex signal receiving systems in which the transfer functions are difficult to obtain. Moreover, P. Orlov et al. presented a unified description of a new approach for contactless detection, identification, and diagnostics of electrical connections and described an idea and principles of using modal probing for these tasks.

Another two studies in this special issue investigated techniques in statistics. H. Sun reported an investigation of stretched exponential function in quantifying long-term memory of extreme events based on artificial data following Lévy stable distribution. It turns out that the stretched exponential distribution provides a reliable way to estimate the scaling behavior of extreme event intervals. In addition, considering the statistic numerical characteristics are often required in the probability-based damage identification and safety assessment of functionally graded material (FGM) structures, Y. Xu et al. presented a stochastic model updatingbased inverse computational method to identify the secondorder statistics (means and variances) of material properties as well as distribution of constituents for damaged FGM structures with material uncertainties.

This special issue also gathers several interdisciplinary studies from other fields. For example, an effective approach is introduced by $\mathrm{H}$. Wei et al. to predict the magnitude of reservoir-triggered earthquake (RTE), based on support vector machines (SVM) and fuzzy support vector machines (FSVM) methods. Both the SVM and FSVM models are found to be effective in the prediction of the magnitude of RTE with high accuracy. Z. Wang et al. reviewed conventional methods for separating the suspended solids from lubricating oils and presented ultrasonic separation methods for particle separation, which is environment-friendly and has a high efficiency. N. F. Alkayem et al. presented a hybrid elitistguided search combining a multiobjective particle swarm optimization, Lévy flights, and the technique for the order of preference by similarity to ideal solution investigated. The proposed method shows good performance even under noisy conditions and in the case of incomplete mode shapes. C. Liu reported a damage detection method for refractory based on principle component analysis and Gaussian mixture model. Two types of damage were utilized to verify the effectiveness of the proposed method. Finally, C. Li proposed an algorithm which is built to obtain the rigorous solution approaching upper and lower bound values simultaneously, satisfying the static boundary and the kinematical boundary based on the slip line field, while stress discontinuity line and velocity discontinuity line are key points.

All in all, this special issue aims to aggregate the latest research efforts contributing to theoretical, methodological, and technological advances in detecting anomalies, forecasting potential degradation, and classifying faults from complex environments and signals. These methods are expected to address the existing challenges for a real-world PHM system.

\section{Conflicts of Interest}

The editors declare that they have no conflicts of interest regarding the publication of this special issue.

Minvydas Ragulskis
Chen Lu
Maosen Cao
Gangbing Song
Rafal Burdzik




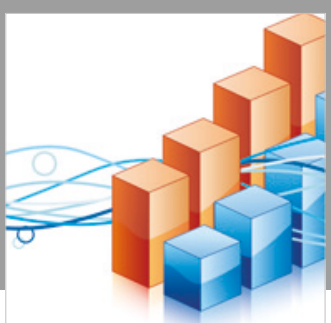

Advances in

Operations Research

\section{-n-m}
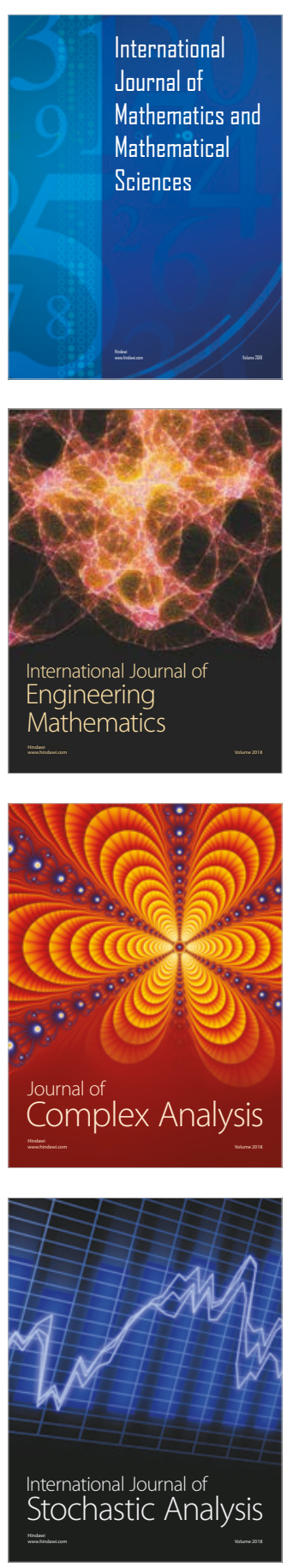
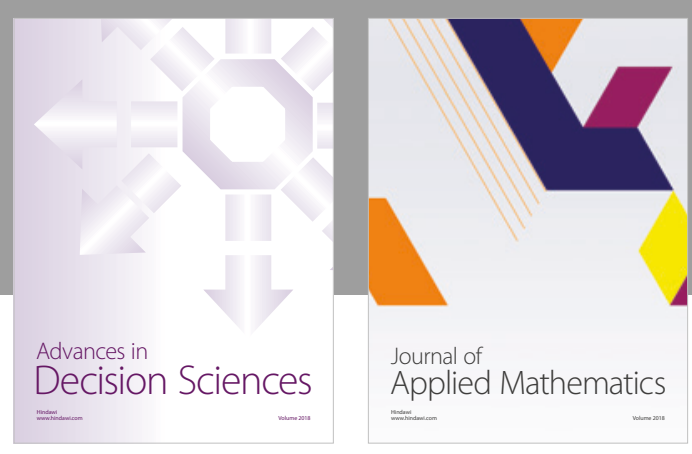

Journal of

Applied Mathematics
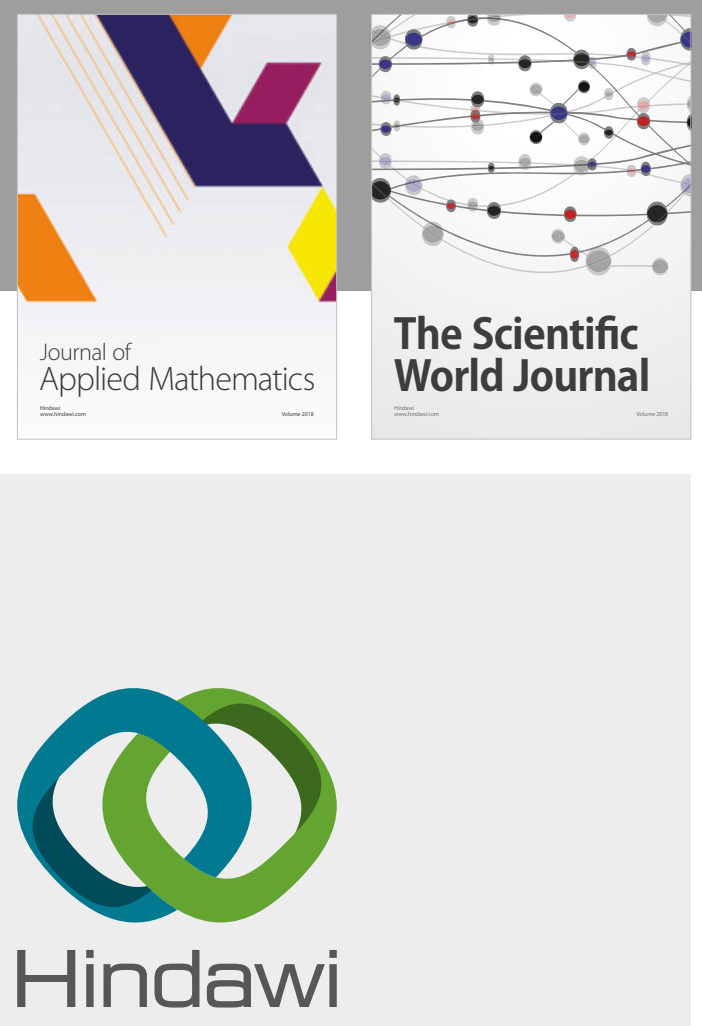

Submit your manuscripts at

www.hindawi.com

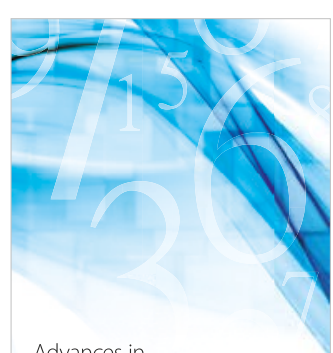

Advances in
Numerical Analysis
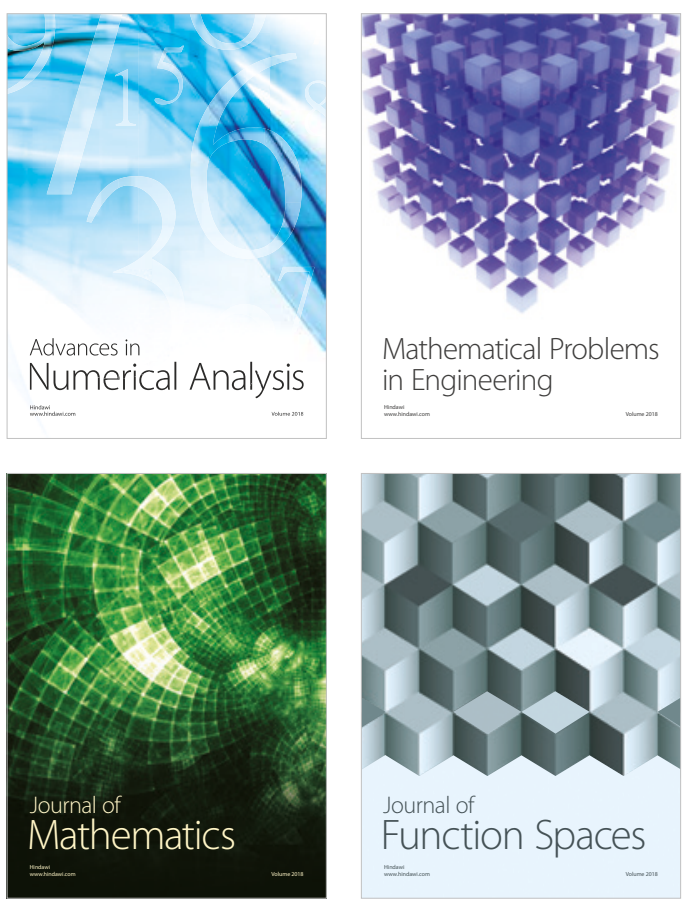

Mathematical Problems in Engineering

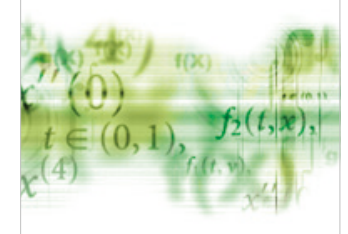

International Journal of

Differential Equations

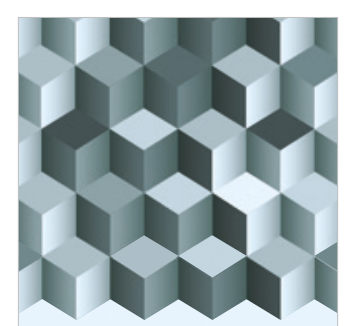

Journal of

Function Spaces
The Scientific

World Journal

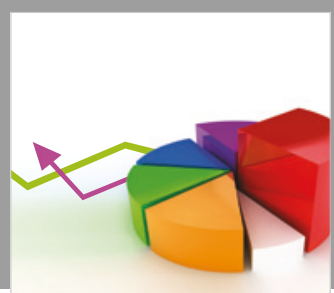

Journal of

Probability and Statistics
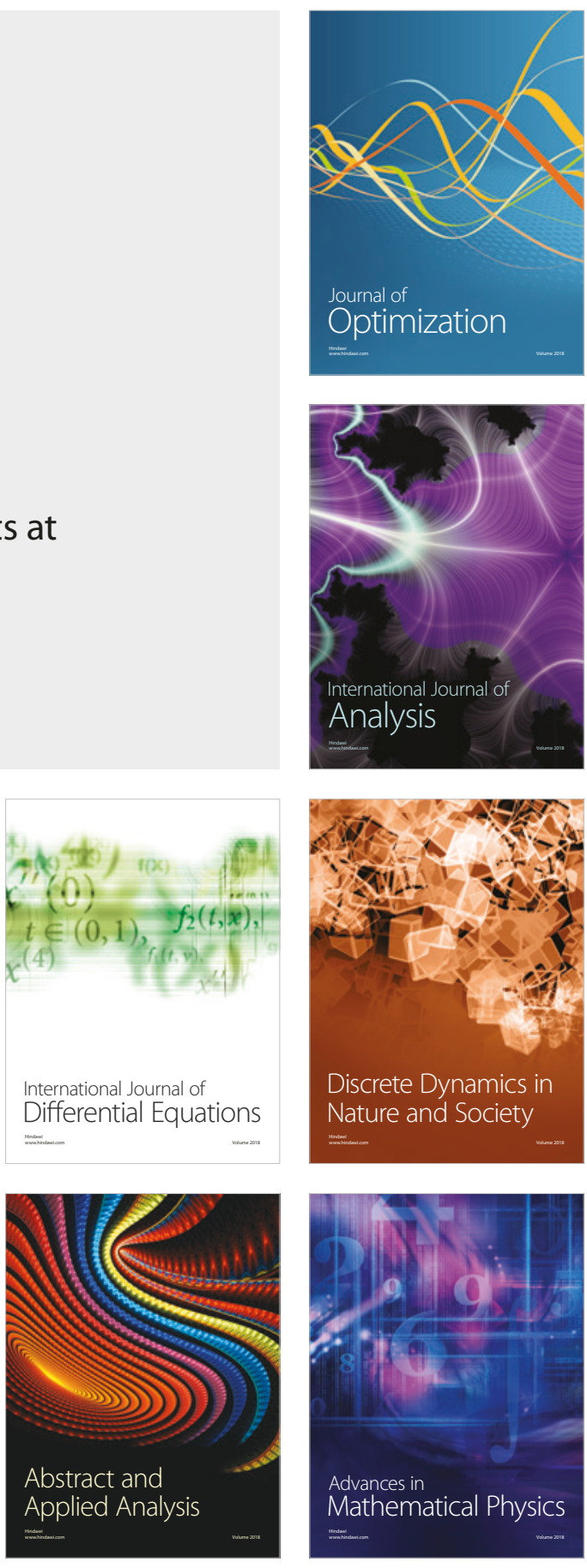